\title{
Appendiceal Diverticulitis
}

\author{
Tho Pham ${ }^{1}$, Shiran Wijeratne ${ }^{2}$
}

${ }^{1}$ The University of Notre Dame Australia, Melbourne Clinical School, Australia; ${ }^{2}$ Mercy Health - Werribee Mercy Hospital, Melbourne, Australia.

\section{Corresponding Author: \\ Dr. Tho Pham \\ Email: thopham13@gmail.com}

This is an Open Access article distributed under the terms of the Creative Commons Attribution License (creativecommons.org/ licenses/by/3.0).

Received Accepted Published

July 7, 2017

November 27, 2017

December 152017

\begin{abstract}
Background: Appendiceal diverticulitis is a rarely diagnosed condition with an incidence of less than $2 \%$ in appendectomy specimens. Although first documented in 1893, recent documented cases have been rare. Case Report: This case report describes a 52 year old man presenting with appendicitis like symptoms, subsequently found to have perforated appendiceal diverticulitis on histopathology. Conclusion: Appendiceal diverticulitis should be considered as a differential in right iliac fossa pain. Progression of appendiceal diverticulitis is often insidious resulting in delayed presentations and subsequently higher incidences of perforation and mortality rates.
\end{abstract}

Keywords: Appendix, Appendectomy, Diverticulum, Diverticulitis, Pain.

\section{Introduction}

Appendiceal diverticulitis (AD) is a rare condition. First described in 1893 by Kelynack [1], recent accounts of the condition have been uncommon. We report a case of $\mathrm{AD}$ in a 52 year old man which we believe to be the first documented case of AD in Australia for over a decade.

\section{Case Report}

A healthy 52-year-old male presented to emergency department with four days of progressive, intermittent abdominal pain originating in the right lower quadrant with no associated fever, nausea, anorexia or emesis. His medical history included diabetes and hypertension. The patient was hypertensive, tachycardic, tachypneic, afebrile and appeared unwell. The abdomen showed signs of right iliac fossa peritonitis. Blood count was normal however, C-reactive protein was elevated. Ultrasound and computed tomography (CT) revealed findings suggestive of ruptured appendicitis [Fig.1,2].
During his laparoscopy, the patient was found to have acute appendicitis with perforation and an appendicectomy with washout was performed. The patient remained well at one week follow up and was discharged under the care of his general practitioner. Subsequent histopathologic examination however, indicated a perforated AD with submucosal/subserosal chronic inflammation and fibroblasts due to acute appendicitis.

\section{Discussion}

$\mathrm{AD}$ is rare with an incidence of $0.004-2 \%$ in appendectomy specimens [2-5]. False diverticula is the more common form and is thought to occur secondary to orifice obstruction and muscular contractions leading to increased intraluminal pressure and inflammation [4,6,7]. Approximately one third of patients with appendiceal diverticulosis will progress to diverticulitis [2].

Both AD and classical appendicitis (CA) may present with right iliac fossa pain, fever and leucocytosis [7]. However, AD has a distinct 


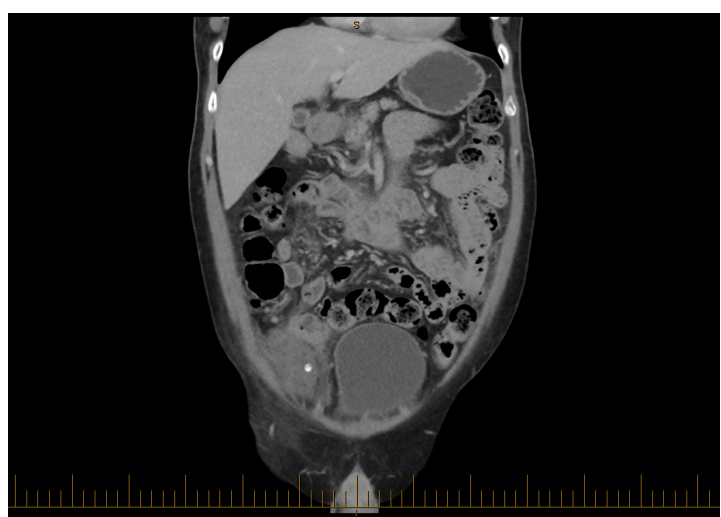

Fig.1: CT abdomen, coronal view showing appendicitis with inflammatory collection and appendicolith.

pathophysiology that manifests atypically to CA, presenting more commonly in males and people with cystic fibrosis [3-5,7]. AD usually affects the older population (average 38.8 years) compared to CA [2-4]. In addition, pain typically develops initially in the RIF without the typical presentation of peri-umbilical pain [7]. Typical gastrointestinal symptoms of CA (e.g. nausea, anorexia, vomiting) are rare in $\mathrm{AD}[2,5]$. This atypical appearance fits with our patient's presentation. Therefore, AD should be a differential, especially in older adult males with abnormal abdominal symptoms.

The vague presentation of $\mathrm{AD}$ can make pre-operative diagnosis difficult possibly leading to delayed presentation, recovery and more complications [5,7]. Appendix perforations are four times more common in patients with $\mathrm{AD}$ compared with CA. Consequently, mortality rates are thirtyfold compared to CA $[2,5]$. Other complications include obstruction and appendiceal mucinous neoplasms associated with pseudomyxoma peritonei $[2,5]$.

There is no established diagnostic radiographic test for $\mathrm{AD}$ but recent studies suggest CT is effective in pre-operative diagnosis $[4,8]$. In our case, CT scan incorrectly identified CA. This may be because the radiological picture of diverticular and CA do not differ significantly [5].

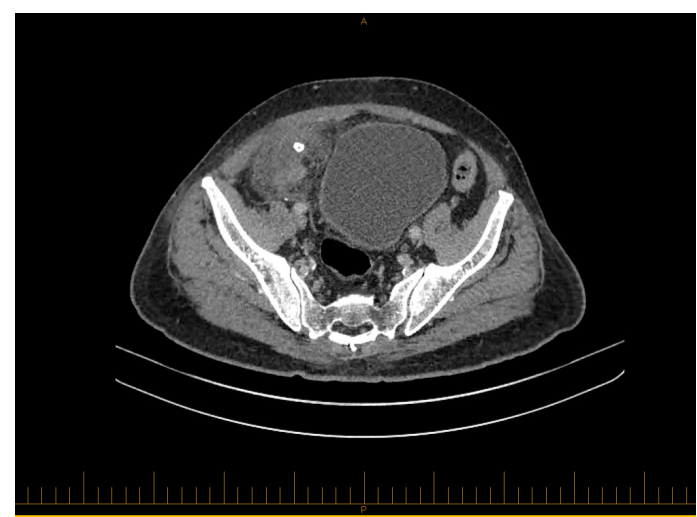

Fig.2: CT abdomen, axial view showing appendicitis with inflammatory collection and appendicolith.

\section{Conclusion}

$\mathrm{AD}$ is rarely diagnosed. Although treatment is similar to $\mathrm{CA}$ [7], AD is a distinct clinical entity and should be considered as a separate differential in diagnosing right iliac fossa pain, especially in older males with atypical abdominal presentations. Progression of AD is insidious; patients often seek medical treatment later than CA leading to higher incidences of perforation and therefore increased mortality rates. AD may also be associated with pseudomyxoma peritonei requiring histological examination of the appendix specimen to rule out complications.

Acknowledgements: We would like to thank Dr Leon Winata, Werribee Mercy Hospital, and Dr Chilan Nguyen, University of Notre Dame Australia, for all their assistance in preparing this case report for publication.

Contributors: TP and SW both contributed to the design of the case report. TP drafted the manuscript with revisions done by SW. TP will act as guarantor. Both authors approved the final version of this manuscript.

Funding: None; Competing interests: None stated.

\section{References}

1. Kelynack TN. A contribution to the pathology of the vermiform appendix: HK Lewis; 1893.

2. Mahmood RD. Appendiceal diverticulosis. BMJ Case Reports. 2010;2010.

3. Friedlich M, Malik N, Lecompte M, Ayroud Y. Diverticulitis of the appendix. Canadian Journal of Surgery. 2004;47:146-147. 
4. Place RJ, Simmang CL, Huber Jr P. Appendiceal diverticulitis. Southern Medical Journal. 2000;93:76-79.

5. Abdull Gaffar B. Diverticulosis and diverticulitis of the appendix. International Journal of Surgical Pathology. 2009; 17:231-237.

6. Käser SA, Willi N, Maurer CA. Prevalence and clinical implications of diverticulosis of the vermiform appendix. Journal of International Medical Research.
2013;41:1350-1356.

7. Phillips BJ, Perry CW. Appendiceal diverticulitis. Mayo Clinic Proceedings. 1999;74:890-892.

8. Osada H, Ohno H, Saiga K, Watanabe W, Okada T, Honda N. Appendiceal diverticulitis: multidetector CT features. Japanese Journal of Radiology. 2012;30:242248. 